\title{
Edge-channel interference controlled by Landau level filling
}

\author{
L. V. Litvin, A. Helzel, H.-P. Tranitz, W. Wegscheider, and C. Strunk \\ Institut für experimentelle und angewandte Physik, Universität Regensburg, D-93040 Regensburg, Germany
}

(Received 9 May 2008; published 5 August 2008)

\begin{abstract}
We study the visibility of Aharonov-Bohm interference in an electronic Mach-Zehnder interferometer in the integer quantum Hall regime. The visibility is controlled by the filling factor $\nu$ and is observed only between $\nu \approx 2.0$ and 1.0 , with an unexpected maximum near $\nu=1.5$. Three energy scales extracted from the temperature and voltage dependences of the visibility change in a very similar way with the filling factor, indicating that the different aspects of the interference depend sensitively on the local structure of the compressible and incompressible strips forming the quantum Hall edge channels.
\end{abstract}

DOI: 10.1103/PhysRevB.78.075303

PACS number(s): 73.23.Ad, 73.63.Nm, 71.10.Pm

\section{INTRODUCTION}

The electronic Mach-Zehnder interferometer ${ }^{1}$ (MZI) was proposed to study the decoherence ${ }^{1,2}$ and orbital entanglement effects ${ }^{3,4}$ by using edge channels in the regime of the quantum Hall effect (QHE). Its high interference contrast, observed at temperature of about $20 \mathrm{mK}$, is the consequence of ballistic transport through the quasi-one-dimensional edge channels. The effect of temperature, ${ }^{1,5,6}$ bias voltage, ${ }^{1,5,7}$ and the interferometer size ${ }^{6,8}$ on the interference contrast are currently under intense investigation. The magnetic field $B$ is another important parameter, which controls the structure of the edge state consisting of compressible and incompressible stripes. ${ }^{9}$ In previous experiments both a monotonic growth of visibility with increasing magnetic field ${ }^{8}$ and a local maximum ${ }^{6}$ were observed within the $\nu=2$ plateau. In these works only the number of edge channels rather than the precise value of the filling factor $\nu=n h / e B$ was specified. Here, $n$ is the electron density, $h$ the Planck constant, and $e$ the elementary charge. In this paper, we systematically study the behavior of the MZI visibility in a broad range of filling factors. We found that the interference for the lowest Landau level appears at a filling factor of $\nu=2.0$, reaches a maximum of visibility of about $50 \%$ at $\nu=1.5$, and then decays to zero near $\nu=1.0$. Although the interference occurs only in the outer edge channel, the visibility is strongly affected by the presence of the inner edge channel and its evolution, when $\nu$ is varied between 1 and 2 .

\section{EXPERIMENTAL DETAILS}

The interferometer (see Fig. 1) was fabricated on the basis of a modulation doped $\mathrm{GaAs} / \mathrm{Ga}_{x} \mathrm{Al}_{1-x} \mathrm{As}$ heterostructure containing a two-dimensional electron gas (2DEG) $90 \mathrm{~nm}$ below the surface. At $4 \mathrm{~K}$, the unpatterned 2DEG density and mobility were $n=2.0 \times 10^{15} \mathrm{~m}^{-2}$ and $\mu=206 \mathrm{~m}^{2} /(\mathrm{Vs})$, respectively. Photolithography was employed to define Hall bars with large contacts (connected to leads $S$, D2, and all gates at Fig. 1). The ring-shaped interferometer mesa, contact D1, the quantum point contacts (with air bridges), and the modulation gate (labeled as MG in Fig. 1) were patterned by means of electron-beam lithography. The quantum point contact (QPC) No. 0 was used to select the outer edge channel for the interference experiment. We studied two MZIs with an arm length of 14 and $9 \mu \mathrm{m}$ and an arm width of 2.5-3 $\mu \mathrm{m}(1.7 \mu \mathrm{m})$, respectively (see Fig. 1). The QPCs of the larger MZI had $120 \mathrm{~nm}$ gap between sharp tips (tip radius $\approx 50 \mathrm{~nm}$ ); for the smaller MZI a $400 \mathrm{~nm}$ gap was used. The area between two interfering paths, determined from the period of the $\mathrm{AB}$ oscillations in a magnetic field, was 48 and $25 \mu \mathrm{m}^{2}$ for these MZIs. A standard lock-in technique $(f$ $\sim 300 \mathrm{~Hz}$ ) with $1 \mu \mathrm{V}$ excitation at terminal $S$ and detection at terminal D2 was employed (see Fig. 1). Most of the measurements were performed at a temperature of $25 \mathrm{mK}$.

\section{MAGNETIC-FIELD DEPENDENCE OF THE VISIBILITY}

In Fig. 2 (inset) we show a typical trace of current $I\left(V_{\mathrm{MG}}\right)$ in detector D2 vs the voltage at the modulation gate $V_{\mathrm{MG}}$. The measured interference contrast is quantified by the visibility which is defined as $\nu_{I}=\left(I_{\max }-I_{\min }\right) /\left(I_{\max }+I_{\min }\right)$. When changing magnetic field $B$ the QPC transmission has to be readjusted to $1 / 2$, because it sensitivitely depends on $B$. In some cases resonances in the QPC transmission characteristics occurred. In this case the half transmission point with the highest $\nu_{I}$ was selected. ${ }^{10}$ In order to relate the magnetic field to the filling factor in the MZI arms, the two-point conductance of the interferometer between terminal $S$ and D2 with all QPCs opened was measured [Fig. 2(a)]. The electron density of the narrowest section of the interferometer can be

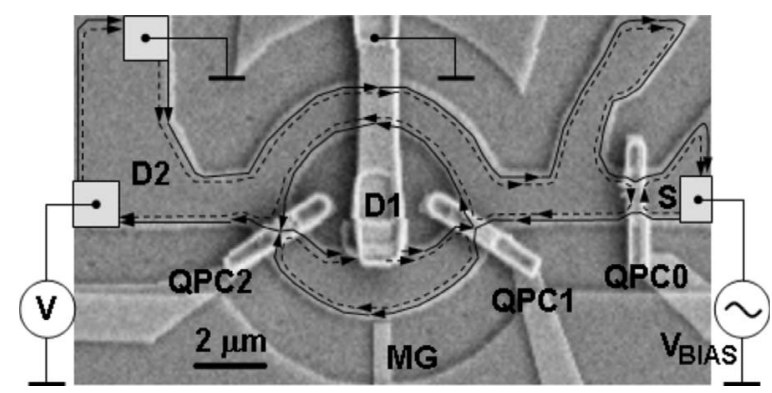

FIG. 1. SEM image of smaller Mach-Zehnder interferometer with the scheme of edge states for filling factor 2. The transmissions of QPC1 and QPC2 are set to 0.5. QPC0 reflects the inner edge channel (dashed line) and transmits the outer one (solid line). MG shifts the phase. 


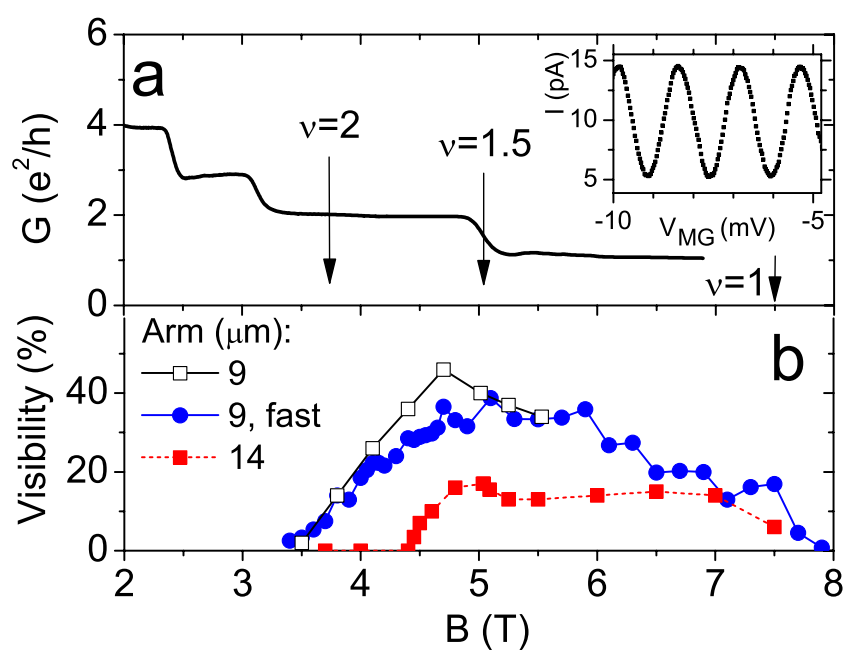

FIG. 2. (Color online) (a) Two terminal magnetoconductance of the large interferometer with all QPCs opened. Inset shows an example of interference pattern at $B=4.7 \mathrm{~T}$. (b) Visibility versus magnetic field for both interferometers. Full and open squares correspond to data acquired with at least $4 \mathrm{~h}$ waiting time, full circles with $1 \mathrm{~h}$.

probed in this way. For the larger interferometer the transition between the $\nu=2$ and $\nu=1$ Hall plateaus is located at $B=5 \mathrm{~T}$ and corresponds to the filling factor $\nu=1.5$. For the smaller interferometer the transition point shifts to $4.8 \mathrm{~T}$. These reference points were used to determine the filling factor in the range of $3 \mathrm{~T}<B<8 \mathrm{~T}$. The visibility of the larger MZI shown in Fig. 2(b) with full squares emerges at $B \gtrsim 4.4 \mathrm{~T}(\nu \approx 1.7)$, reaches a maximum of $17 \%$ at $\nu=1.5$, and then nonmonotonically decreases to $6 \%$ at $B=7.5 \mathrm{~T}(\nu$ $=1.0$ ). For the smaller interferometer, a measurable visibility [full circles and open squares at Fig. 2(b)] emerges near $B$ $=3.5 \mathrm{~T}(\nu \approx 2.0)$, reaches maximum around $B=5 \mathrm{~T}$, and then decreases to zero at about $B=8 \mathrm{~T}(\nu \approx 0.9)$. Right after the magnetic field is ramped to the next data point, the visibility drops significantly (about 50\%). However, after some waiting time it recovers and approaches a saturation value. The saturation takes up to $10 \mathrm{~h}$. As a reasonable compromise between optimum visibility and reasonable data-acquisition time, we have chosen $1 \mathrm{~h}$ [full circles in Fig. 2(b)] and $4 \mathrm{~h}$ [open squares in Fig. 2(b)] for the waiting time. A measurable visibility was observed in the $\nu$ interval $1.0 \leqq \nu \leqq 2.0$ with a maximum in $\nu_{I}(B)$ near $(\nu=1.5)$.

To assure that it is the filling factor which controls the visibility and not the absolute magnetic-field magnitude, we changed the electron density in the interferometer by a back gate. A decrease in the electron density shifts the entire $\nu_{I}(B)$ curve to smaller magnetic fields [Fig. 3(a) and inset in Fig. 3(b)]. As an independent check of the determination of filling factor within the interferometer arm, we looked at the backscattering properties. The quantum point contact QPC0 is set to transmit only the outer channel, QPC1 is completely closed, and QPC2 is completely opened. In absence of backscattering within the lower interferometer arm, QPC1 redirects all current from $S$ to the detector D1, while D2 sees zero signal. However, when the filling factor is close to 1.5, backscattering occurs between the counter-propagating lower

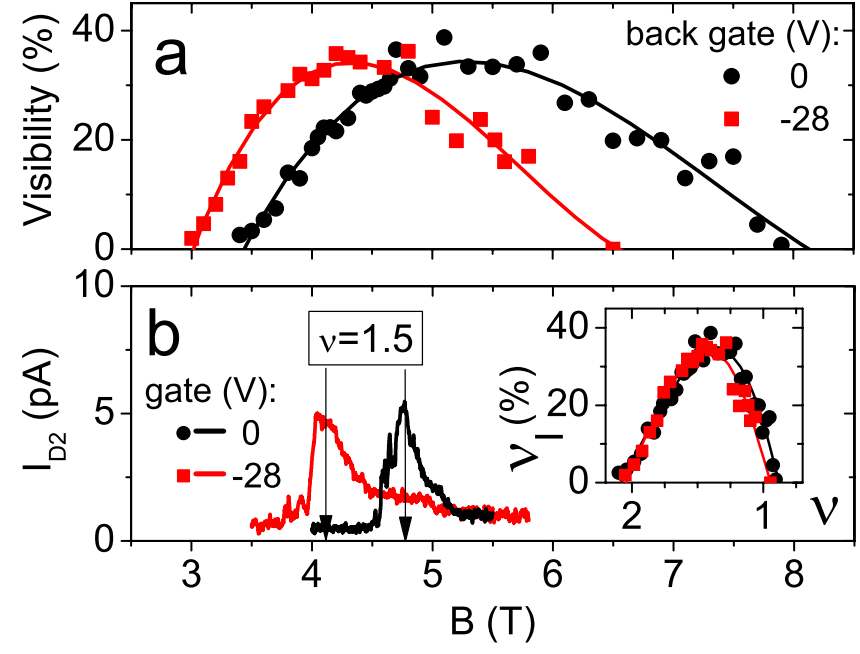

FIG. 3. (Color online) (a) Visibility versus magnetic field for two back gate voltages, i.e., electron densities. (b) Determination of the $\nu=1.5$ point. Current recorded at detector D2, when QPC1 is closed and QPC2 is opened. The peak in each curve corresponds to maximum scattering between edges in the lower MZI arm and correspond to $\nu=1.5$. Inset: Data from (a) plotted vs filling factor.

arm edge channels which appears as signal in D2 [Fig. 3(b)]. The maximum of this signal corresponds to the point $\nu$ $=1.5$ where the backscattering is strongest. We see that a back gate voltage of $-28 \mathrm{~V}$ shifts the peak, in other words, the point of maximum scattering, from 4.8 to $4.1 \mathrm{~T}$, i.e., the density decreases by $15 \%$. The maximum of visibility was earlier reported to occur on the upper end of the $\nu=2$ plateau, ${ }^{6}$ while we observed it at $\nu=1.5$. This discrepancy may be caused by an unequal filling factor in the two MZI arms in Ref. 6. According to Ref. 9 a mesa width which is not much larger than the depletion length results in different electron densities and therefore different filling factors. To avoid this problem we used the same width for both interferometer arms as well as for the input and output leads.

\section{CHARACTERISTIC ENERGY SCALES}

There are three fundamental reasons for a reduction of the visibility: (i) genuine decoherence by inelastic scattering; (ii) phase averaging, due to a finite-energy window, imposed by temperature and voltage; ${ }^{11}$ and (iii) phase averaging due to fluctuations of charges trapped nearby. ${ }^{2}$ The last possibility can probably be excluded, since fluctuations in the environment are not expected to strongly depend on $B$. A recent experimental study of $\nu_{I}(T, B)$ showed that it changes exponentially with $T$ :

$$
\nu_{I}=\nu_{I 0} \exp \left(-T / T_{0}\right)=\nu_{I 0} \exp \left(-2 L / l_{\varphi}\right),
$$

where the characteristic temperature $T_{0}$ is inversely proportional to the length of the interferometer arm $L$, i.e., $T_{0}$ $\propto l_{\varphi} T / 2 L$ or $l_{\varphi} \propto 2 L T_{0} / T{ }^{6}$ The electromagnetic environment of the interferometer is expected to give rise to a $l_{\varphi} \propto 1 / T$ dependence. $^{12}$ On the other hand, it is again unclear why such an environmental effect should strongly vary with $B$.

As demonstrated by the solid lines in Fig. 4, our data also vary exponentially with $T$ above $45 \mathrm{mK}$. However, at lower 


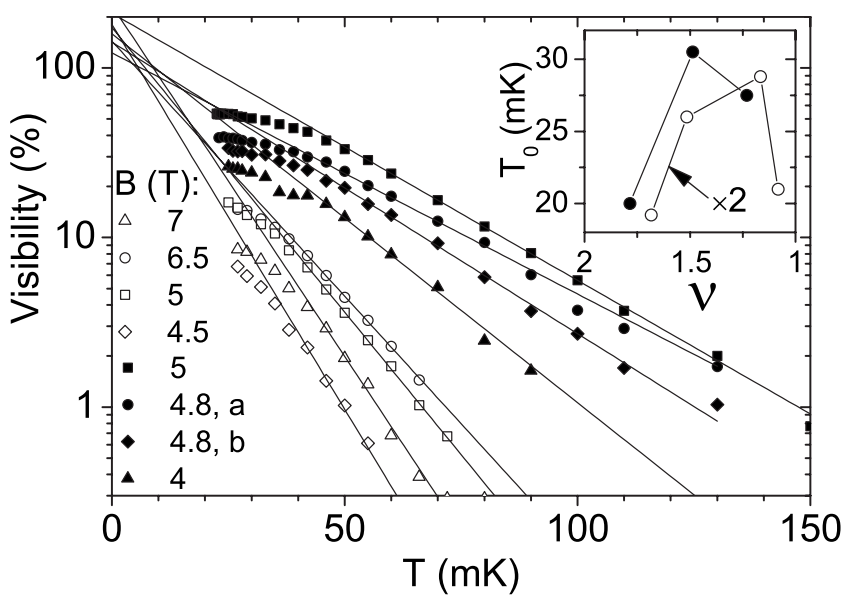

FIG. 4. (a) Temperature dependencies of visibilities for large (open symbols) and small (full symbols) MZIs at different magnetic fields. The curves "a" and " $\mathrm{b}$ " for $B=4.8 \mathrm{~T}$ correspond to different QPC half transmission points. Inset: Characteristic temperatures $T_{0}$ extracted from the exponential fits according to Eq. (1). The data for the large interferometer (open circles) have been multiplied by 2 .

temperatures a crossover to a weaker temperature dependence is observed. The presence of such a crossover is reflected by the extrapolated values of $\nu_{I 0}$, which significantly exceed the allowed maximum of $100 \%$, and the fact that the fit lines do not cross at $T=0$, but rather at $7 \mathrm{mK}$. Although it is notoriously hard to exclude that electron heating contributes to the apparent saturation of $\nu_{I}$ at $T \lesssim 45 \mathrm{mK}$ (Fig. 4), the latter two facts refer to the high-temperature regime and indicate that the behavior of $\nu_{I}(T)$ may be more complex than a simple exponential.

Despite these differences, our data confirm a clear correlation between the extracted values of $T_{0}$ and the visibility, when the magnetic field is varied. Like the visibility, $T_{0}$ has a maximum around $\nu=1.5$ (see the inset of Fig. 4). ${ }^{13}$ Thus, it is clear that the phase coherence length is magnetic field dependent.

The visibility can also be measured as a function of a dc voltage added to the small ac bias. This differential visibility shows a lobe structure ${ }^{7,8}$ vs $V_{\mathrm{dc}}$. In Fig. 5(a) we show the evolution of the lobe structure with filling factor. The observed change of the lobe characteristics resembles the change of visibility with $\nu$ [see Fig. 2(b)], i.e., the largest distance between the zeros in $\nu_{I}$ is found near $\nu=1.5$ and reduces, when $\nu$ moves from $=1.5$ to $\nu=1.0$ or 2.0 [Fig. 5 (b), open squares]. For $\nu>1.5$ more than one pair of side lobes can be observed [Fig. 5(a), B=3.9 T]. The data in Fig. 5(a) can be well approximated by a product of an oscillatory function and a Gaussian envelope: $\nu_{I}$ $=\nu_{I 0}\left|\cos \left(\pi \mathrm{eV} / \varepsilon_{L}\right)\right| \exp \left[-(\mathrm{eV})^{2} / 2 \varepsilon_{0}^{2}\right]$ which contains the parameters $\varepsilon_{L}$ as period of the cosine term and $\varepsilon_{0}$ as characteristic width of the envelope. For a direct comparison, we plot these characteristic energies together with the characteristic temperature $k_{B} T_{0}$ extracted from the temperature dependence of $\nu_{I}$. The energies $\varepsilon_{L}$ and $\varepsilon_{0}$ agree rather well for $2>\nu$ $>1.5$, while $\varepsilon_{0}$ is slightly smaller than $\epsilon_{L}$ for $\nu<1.5$. On the other hand, $k_{B} T_{0}$ is about 15 times smaller. Despite the difference in numbers, the overall $\nu$ dependence of all energy
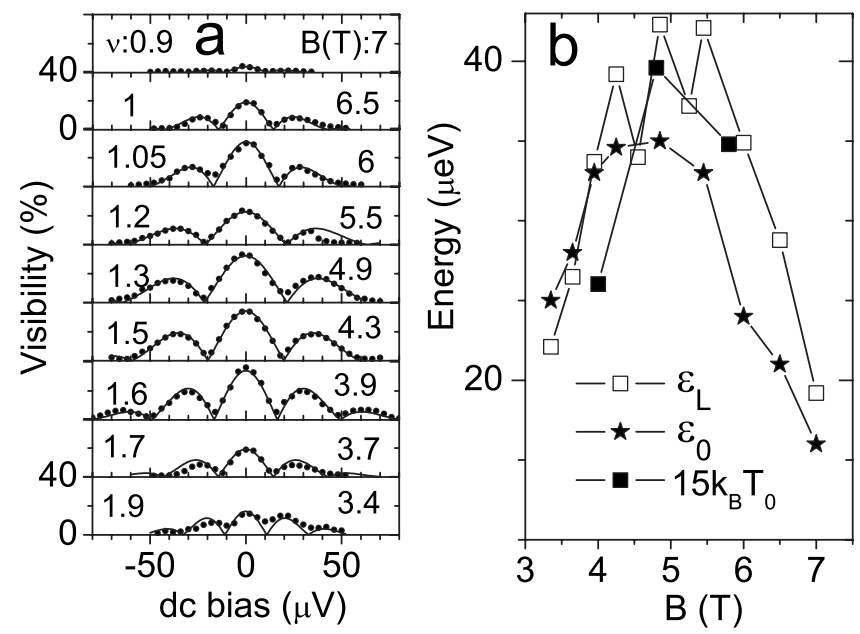

FIG. 5. Differential visibility of the small MZI. (a) Lobe structures at different magnetic fields at $V_{\mathrm{BG}}=-25 \mathrm{~V}$. (b) Characteristic energies $\varepsilon_{L}$ and $\varepsilon_{0}$ (see text) extracted from $\nu_{I}(V)$ together with $k_{B} T_{0}$ as a function of magnetic field $B$.

scales is quite similar. Hence, it appears that the $B$ dependence of the characteristic energies is closely related to the evolution of the structure of edge states with $\nu$.

\section{DISCUSSION}

For the following, one has to keep in mind that QPC0 is tuned such that current is injected exclusively into the outer incompressible strip with $\nu=1$. According to accepted theory, ${ }^{14}$ at small bias the current flows close to the interface between compressible and incompressible stripes. Already in Ref. 8 it was realized that the lobe pattern cannot be understood in terms of a single electron picture, nor within a simple mean-field approach. Nevertheless, the charges injected into the interferometer by the dc component of the bias appear to induce an overall phase shift, i.e., they act similar to the modulation gate. Youn et $a l .{ }^{15}$ proposed that the average phase shift $\langle\delta\rangle$ is determined by the average number $N$ of nonequilibrium electrons and the intrachannel Coulomb interaction constant $U_{0}$ :

$$
\langle\delta\rangle=N \frac{U_{0} t_{f l}}{\hbar}=\frac{|e| V t_{f l}}{\hbar} \frac{U_{0} t_{f l}}{\hbar},
$$

where $t_{f l}=L / v_{D}$ is the traversal time of the electrons through the interferometer and $v_{D}$ the drift velocity along the edge. Using $v_{D} \approx 3 \cdot 10^{4} \mathrm{~m} / \mathrm{s}$ and $L=9 \mu \mathrm{m}$, one obtains $N \approx 1$ at $V_{\mathrm{dc}}=10 \mu \mathrm{V}$. With increasing $N \propto V_{\mathrm{dc}}$ the number of possible charge-density distributions in the interferometer and hence the fluctuations of $\delta$ increases, which according to the numerical calculations in Ref. 15 leads to a suppression of the visibility at higher $V_{\mathrm{dc}}$. For smaller $U_{0}$ a larger $V_{\mathrm{dc}}$ is needed to reach the first zero of $\nu_{I}$.

If one now assumes that the interaction parameter $U_{0}$ is affected by the screening properties of the environment of the outer edge channel, the changes of the structure of the 2DEG enclosed by the outer edge between $\nu=2$ and $\nu=1$ would indeed suggest an nonmonotonic variation of $U_{0}(B)$, 
because the screening is most effective at $\nu=1.5$, where the entire bulk of the 2DEG is compressible. On the other hand, at $\nu=1$ and 2, the bulk of the 2DEG is incompressible, implying reduced screening and correspondingly larger values of $U_{0}$. This scenario is consistent with the observed nonmonotonic variation of $\varepsilon_{L}$. The energy scales $\varepsilon_{L}$ and $\varepsilon_{0}$ must then be related to $\hbar / t_{f l}$ and $U_{0}(B)$.

Very recently, another theory based on the chiral Luttinger liquid approach to the QHE has been suggested. ${ }^{16}$ In this theory, the excitations are dipolar (neutral) and charged edge magnetoplasmon modes with different group velocities $v$ and $u$. The model allows to calculate $l_{\varphi}(T)=\hbar u v / \pi k_{B} T(u-v)$, which is consistent with the observed $T$ dependence of $l_{\varphi}$. In addition it predicts for the ratio between $\epsilon_{L} / k_{B} T_{0}=2 \pi^{2}$ $\simeq$ 19.7. From our data we deduce a similar experimental value of $\epsilon_{L} / k_{B} T_{0} \simeq 15$. This model requires two well defined edge channels and is valid at $\nu \geq 1.5$. Hence, it remains to be explained, why the experimental data show a visibility maximum for $\nu \lesssim 1.5$.

Another theory for $\nu=1$ results in a $l_{\varphi} \propto T^{-3}$ for screened and $l_{\varphi} \propto T^{-1} \ln ^{2} T$ for unscreened Coulomb interactions. ${ }^{17}$ For the transition between $\nu=1$ and $\nu=2$, so far no theory exists. Qualitatively, one may expect that a fermionic picture is more appropriate here, since the screening by the compressible interior of the interior of the 2DEG tends to suppress the Luttinger liquid effects.

For a better microscopic understanding of the effect, it is essential to relate the various phenomenological energy scales of the different theories to a realistic model of the structure of the edge channels, i.e., the distribution of compressible and incompressible strips at the mesa edge. ${ }^{18}$ This distribution considerably changes between $\nu=1$ and 2. At $\nu$ $=1.5$ and above the outer incompressible strip is well localized at the mesa edge. Depending on the steepness of the confining potential, it starts to spread out for $\nu \lesssim 1.5$ and fills the whole mesa, once $\nu=1$ is reached. The precise reason for the decay of the interference for $\nu<1.5$ is still an open question. The measured temperature dependence of the visibility suggests that $l_{\varphi}$ is suppressed in this regime again. On the other hand, an edge strip that spreads out over $100 \mathrm{~nm}$ and more cannot be considered as a quasi-one-dimensional object anymore. In particular, it does not enclose a well defined magnetic flux. The integration of such QHE-specific features into models considering simple one-dimensional conduction channels provides new challenges for the theory.

\section{CONCLUSIONS}

In summary we investigated the effect of the filling factor on the visibility in a Mach-Zehnder interferometer. Surprisingly, the visibility was found to be highest around $\nu=1.5$ and decreases to zero when the adjacent integer filling factors are reached. This dependence originates from an evolution of the structure of edge channels with magnetic field, which strongly affects three energy scales of the interference in a very similar way: $k_{B} T_{0}$, which determines the temperature dependence of the visibility in the linear regime, and $\epsilon_{L}$ and $\epsilon_{0}$, which determine the size and damping of the lobe structure of $\nu_{I}$ in the nonlinear regime. This observation suggests that the linear and the nonlinear regimes are governed by the same energy scale.

\section{ACKNOWLEDGMENTS}

We thank M. Heiblum and I. Neder for support in the framework of EU-Transnational Access Program, Contract No. RITA-CT-2003-506095. We are grateful to A. Siddiki, S. Ludwig, E.V. Devyatov, E. Sukhorukov, and M. Büttiker for helpful discussions. The work was funded by the DFG within the SFB631 "Solid state quantum information processing" and the BMBF via Project No. 01BM465 within the program "Nanoquit."
${ }^{1}$ Y. Ji, Y. Chung, D. Sprinzak, M. Heiblum, D. Mahalu, and H. Shtrikman, Nature (London) 422, 415 (2003).

${ }^{2}$ F. Marquardt and C. Bruder, Phys. Rev. B 70, 125305 (2004).

${ }^{3}$ P. Samuelsson, E. V. Sukhorukov, and M. Büttiker, Phys. Rev. Lett. 92, 026805 (2004).

${ }^{4}$ I. Neder, N. Ofek, Y. Chung, M. Heiblum, D. Mahalu, and V. Umansky, Nature (London) 448, 333 (2007).

${ }^{5}$ L. V. Litvin, H.-P. Tranitz, W. Wegscheider, and C. Strunk, Phys. Rev. B 75, 033315 (2007).

${ }^{6}$ P. Roulleau, F. Portier, D. C. Glattli, P. Roche, A. Cavanna, G. Faini, U. Gennser, and D. Mailly, Phys. Rev. Lett. 100, 126802 (2008).

${ }^{7}$ P. Roulleau, F. Portier, D. C. Glattli, P. Roche, A. Cavanna, G. Faini, U. Gennser, and D. Mailly, Phys. Rev. B 76, 161309(R) (2007).

${ }^{8}$ I. Neder, M. Heiblum, Y. Levinson, D. Mahalu, and V. Umansky, Phys. Rev. Lett. 96, 016804 (2006).

${ }^{9}$ D. B. Chklovskii, K. A. Matveev, and B. I. Shklovskii, Phys.
Rev. B 47, 12605 (1993).

${ }^{10}$ L. V. Litvin, A. Helzel, H.-P. Tranitz, W. Wegscheider, and C. Strunk, Physica E (Amsterdam) 40, 1706 (2008).

${ }^{11}$ V. S.-W. Chung, P. Samuelsson, and M. Buttiker, Phys. Rev. B 72, 125320 (2005).

${ }^{12}$ G. Seelig and M. Buttiker, Phys. Rev. B 64, 245313 (2001).

${ }^{13}$ The uppermost trace in Fig. 4 was measured with back gate voltage of $-25 \mathrm{~V}$, corresponding to a reduced electron density.

${ }^{14}$ See, e.g., the reviews in The Quantum Hall Effect, edited by R. E. Prange and S. M. Girvin (Springer-Verlag, New York, 1987); E. V. Devyatov, Phys. Usp. 50, 197 (2007).

${ }^{15}$ S.-C. Youn, H.-W. Lee, and H.-S. Sim, Phys. Rev. Lett. 100, 196807 (2008).

${ }^{16}$ I. P. Levkivskyi and E. V. Sukhorukov, Phys. Rev. B 78, 045322 (2008).

${ }^{17}$ J. T. Chalker, Yuval Gefen, and M. Y. Veillette, Phys. Rev. B 76, 085320 (2007).

${ }^{18}$ A. Siddiki and F. Marquardt, Phys. Rev. B 75, 045325 (2007). 